\title{
A large-scale LED array to support anticipatory driving
}

\author{
Florian Laquai, Fabian Chowanetz and Gerhard Rigoll \\ Institute for Human-Machine-Communication \\ Technische Universität München \\ Theresienstr. 90, 80333 Munich, Germany
}

\begin{abstract}
We present a novel assistance system which supports anticipatory driving by means of fostering early deceleration. Upcoming technologies like Car2X communication provide information about a time interval which is currently uncovered. This information shall be used in the proposed system to inform drivers about future situations which require reduced speed. Such situations include traffic jams, construction sites or speed limits. The HMI is an optical output system based on line arrays of RGBLEDs. Our contribution presents construction details as well as user evaluations. The results show an earlier deceleration of 3.9 - $11.5 \mathrm{~s}$ and a shorter deceleration distance of $2-166 \mathrm{~m}$.
\end{abstract}

Index Terms-LED, anticipatory driving, speed reduction, driver assistance, color calibration, automotive

\section{INTRODUCTION}

Current developments in Car2Car and Car2Infrastrucure communication (Car2X communication) provide a multitude of new data sources that allow for a much more detailed modeling of the vehicle surroundings than today. Unlike in current production systems, where drivers get assistance for long-term maneuver planning on the navigation level or precrash assistance for extreme short-term maneuvers, this gap can be closed in the future with Car2X communication based systems. Most Advanced Driver Assistance Systems (ADAS) in production or research either directly influence vehicle dynamics or display icons and text to inform the driver. As mentioned in an expert study under real driving conditions in [1], a abstract LED display has the potential to alert the driver in an unobtrusive yet explicit way. In the referenced study the LED array was used to support a Night Vision system with directional information about potential collision targets. Compared to the system in [1], the one presented in this paper spans over a larger viewing angle and supports full color output.

Another study (see [4]) uses an array of light dots mounted on the walls of a tunnel to influence the perceived driving speed with an optical illusion. Drivers tended to adjust their actual speed to the speed of the moving light dots. A function like this can be transferred to a system such as the proposed one and the effect can be applied in many more situations than only a tunnel scenario.

These examples show that drivers can be influenced with very abstract graphical outputs. They all have in common that they are noticed and interpreted without explicitly looking at them and consequently can be "read" quickly due to the lack of text or complex symbols.
In the following sections a first prototype and corresponding evaluation results are illustrated. Then the large-scale variant and its color calibration is described together with two assistance concepts and their first evaluation results.

\section{FiRST PROTOTYPE AND EVALUATION}

The assistance function shall be an aid for the driver to be informed about future traffic situations where he has to decelerate. By informing the driver early (up to $20 \mathrm{~s}$ ahead) about situations he potentially cannot see, a more fuel efficient and safer driving style can be fostered. It is triggered by obstacles or traffic signs on the future road course which force the driver to decelerate. A reason for this can be a static traffic jam behind an nonvisible curve, a construction site or a speed limit. We assume that this information can be obtained from electronic maps or Car2X communication. Based on this the algorithm described in [2] calculates the time at which the driver should step off the gas pedal to start coasting so the car is decelerated by the motor drag torque as also described in [3]. If the driver does not react on the generated output in time or if the information about the oncoming situation arrives too late, the algorithm creates an output for a suggested higher deceleration of $-0.2 \mathrm{~g}$ which is a common value for "comfort deceleration". In case this is still not sufficient, a stronger deceleration (-0.4 g, common for emergency braking) is suggested to reach a certain target speed.

The first implementation of a RGB-LED array is depicted in figure 1. It consists of two modules which can only display the same color at a time. This means a single RGB-LED driver can be used which is in our case a Chromoflex controller produced by Xeroflex (http://www.xeroflex.com) with a RS232 serial interface. The modules are mounted besides the steering wheel under the instrument cluster. The suggested deceleration strategy is encoded as follows:

- Coasting: green

- Light braking: yellow

- Strong braking: red

Between different output states of the system a transition time of $1 \mathrm{~s}$ is used to create a linear interpolation between colors. Additionally the oncoming situation is represented by an official traffic sign with a logarithmic distance bar. The logarithmic scale is appropriate as it projects large distances on a small portion of the scale and still maintains a reasonable sensitivity to distance changes at small distances. This element 

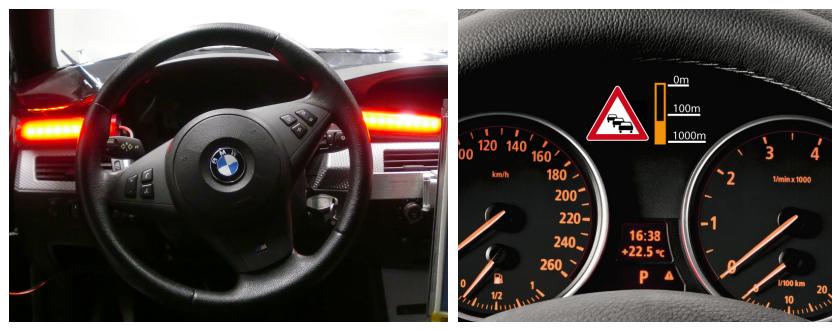

Fig. 1. Left: RGB-LED modules in the cockpit, right: situation representation and distance information

TABLE I

RATINGS OF THE FIRST PROTOTYPE

\begin{tabular}{l|l|l}
\hline Question & Rating & Std. Dev. \\
\hline Would you use this display in your car? & 2.27 & 1.44 \\
\hline Did you find it optically appealing? & 2.33 & 1.45 \\
\hline Could you understand it intuitively? & 3.93 & 1.11 \\
\hline Did it support anticipatory driving? & 3.27 & 1.29 \\
\hline
\end{tabular}

increases user acceptance as it gives an explanation why the system was activated.

\section{A. Experiment description}

In a video experiment all of the above mentioned situations (static traffic jam, construction site, speed limit) occured. The video was recorded from a driving simulator and all relevant data was saved to a log file (speed, distance to speed limitations, assistance output etc.). A special playback software played the video and simultaneously provided the data to the LED system. A group of 30 participants (average age 28.6 years, 16 participants drive more than $5000 \mathrm{~km}$ per year) watched the video and the LED system in a driving simulator mockup.

\section{B. Results}

Participants rated several questions on a scale from 1 (totally disagree) to 5 (totally agree) where a value of 3 indicated a neutral rating. The results shown in table 1 allow the assumption that the system is definitely intutitive since the according rating is higher than the neutral value. The System is not rated as optically appealing and people tend to not wanting to use it in their own cars, though both ratings show low conformity among the participants. The support of anticipatory driving was ranked a bit higher than neutral but the result also has a high standard deviation. Compared to the results in [1], the overall rating was much less positive for this prototype. Participants also had the opportunity to comment positive and negative aspects of the system. On the positive side, 8 participants classified it as "less distracting" and "positively alarming". For the negative aspects, 21 participants stated that the LEDs were "too bright" and "disturbing". Also the chosen color encoding was seen as problematic by users, especially the selection of the color green. Many interpreted this color as a signal meaning "Everything OK" and would not have reacted at all on the system.

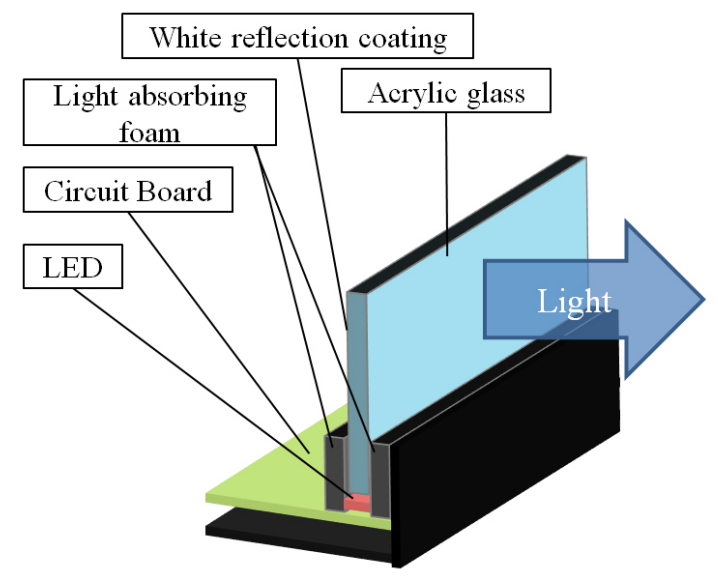

Fig. 2. Assembly of second prototype with indirect lighing (one module)

Consequently the next version of the setup needed the following changes:

- LEDs not pointing directly towards the driver

- more diffuse light instead of distinguishable dots

- addditional experiments regarding light intensity required

- independently controllable LEDs for more sophisticated lighting effects

- larger dimensions of the array for wider support of assistance functions

All of those points were addressed while designing the next version of the system described in the following sections.

\section{LARGE LED ARRAY DESCRIPTION}

The system setup consists of 18 independent LED modules that were designed to be placed next to each other without spacing in between. With each module having a width of $16 \mathrm{~cm}$ the LEDs are arranged at a distance of $1.78 \mathrm{~cm}$ to each other so that a constant density of LEDs per $\mathrm{cm}$ is achieved. Because a diffuse and ambient lighting shall be produced, the diodes must not point directly to the viewer. To accomplish this, the emitted light is being transmitted into a sheet of acrylic glass via the edge of it, see figure 2. The selected LEDs have a maximum luminous intensity of $600 \mathrm{mcd}$ for the red channel, $1400 \mathrm{mcd}$ for the green channel and $400 \mathrm{mcd}$ for the blue channel. The main construction goal is to get an evenly illuminated display area with as few stray light to other directions as possible. Therefore a foam layer on both sides of the glass sheet prevents light from the edge of a LED to escape from the housing. On the backside first a white and then a black coating is applied. The white coating acts as a light refraction layer to obtain light emission mainly on the surface and not on the edges of the sheet. The black coating again works as a measure to prevent any stray light in unwanted directions.

\section{A. Electronical System}

Each module carries 9 RGB-LEDs resulting in 27 individual color channels per module. For the complete system a total of 486 channels have to be adressed to set an illumination value 


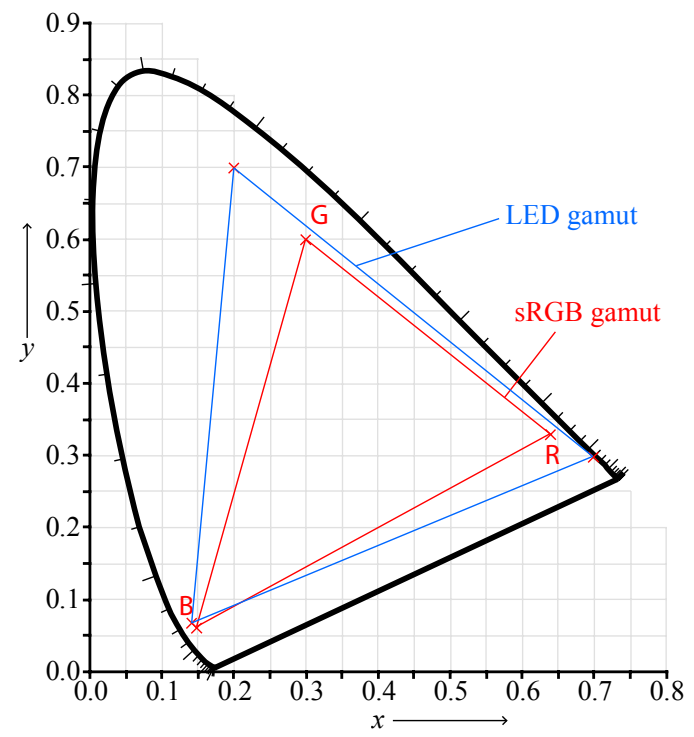

Fig. 3. Comparison of the sRGB gamut (inner triangle) and the LED gamut (outer triangle)

for every channel with a resolution of 256 steps. To handle this amount of channels, a bus system is used for communication. A readily available bus system which meets those requirements and for which affordable hardware exists is the DMX bus. This bus is usually used to control professional lighting installations (see [6]). As the bus system can be run with 3 wires and only 2 additional power supply wires are needed, the wiring effort per module is minimal.

A USB interface is used to connect the DMX bus to a standard PC. On the software side the USB interface is handled by a driver application which provides a TCP socket connection for arbitrary communication partners such as a $\mathrm{C}++$ or Adobe Flash application.

\section{B. Color Calibration}

The LED array will be controlled by a PC and therefore the colors to be displayed are selected on the attached monitor. For this purpose a monitor with sRGB calibration is used. As the color gamut of the LEDs is considerably larger than the sRGB gamut of the monitor (see figure 3) and has different primary colors, the RGB values of the monitor cannot directly be applied. Therefore it is desired to obtain a Matrix $M$ that solves the equation $c_{L E D}=M \cdot c_{s R G B}$ with $c_{L E D}$ and $c_{s R G B}$ as the respective color vectors.

This means a calibration must be conducted to achieve comparable color results from the LED modules and a PC monitor by calculating the matrix $M$. For this purpose a calibration application was developed which displays five reference colors (red, green, blue, orange, yellow) to the participants. The initial color displayed by the module was the uncalibrated RGB value. A module was located directly in front of the monitor to provide the possibility to view both color fields simultaneously as depicted in figure 4 . For every single one

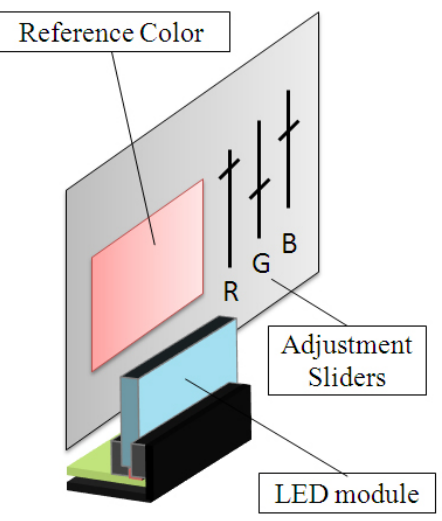

Fig. 4. Setup with one module and calibration application on a sRGB monitor

of the 20 participants the reference colors were slightly varied to accumulate a larger number of calibrated color pairs. Each participant had to adjust the color of the LED module with the three RGB sliders in the calibration application to match the reference as closely as possible.

As the brightness of the module is considerably higher than of the monitor, the task was complicated additionally for the test persons. From the 100 color pairs a transformation matrix $M$ was found using the Least Squares Algorithm which resulted in a relative residual of 0.31 .

$$
M=\left[\begin{array}{ccc}
0.986 & -0.074 & -0.134 \\
-0.074 & 0.97 & -0.277 \\
-0.105 & -0.106 & 0.816
\end{array}\right]
$$

To improve the result of this step and obtain a lower relative residual from the optimization process, all color adjustments from users were normalized to have the same grey value of the reference color. With these normalized color pairs the new transformation matrix $M_{\text {norm }}$ can be obtained with a relative residual of 0.13 :

$$
M_{\text {norm }}=\left[\begin{array}{ccc}
1.173 & -0.123 & -0.152 \\
-0.171 & 1.246 & -0.33 \\
-0.179 & -0.187 & 1.345
\end{array}\right]
$$

This matrix finally showed the desired comparable colors for the monitor and the LED modules. It can be seen that a $c_{S R G B}=(255,0,0)^{T}$ would produce a vector $c_{L E D}=$ $(299,-44,-46)^{T}$ which would be out of the allowed range of $[0,255]$ per color channel. In such cases, also if negative values occur, the respective elements of the vector $c_{L E D}$ are set to the closest interval borders to obtain a valid color vector.

\section{AsSistance CONCEPTS}

As mentioned in the introduction, several experiments with comparable setups or transferrable effects have been conducted by other projects. One concept to be transferred is the speed guiding effect from [4], the other is the idea of a static color changing light dot from the first prototype which will be pursued as a second concept. Both concepts have in common that the reason for a system activation is displayed in the 




Fig. 5. Construction of the Head-Up Display with a standard projector and acrylic combiner screen

Head-Up Display (HUD), see figure 5. For this purpose the same content as in the first prototype (traffic sign and distance indicator) is shown on the HUD. In the first prototype this was done in the instrument cluster between the speed gauge and the RPM gauge (see figure 1 right). As the LED arrays are moved closer to the primary field of view the same position shift is applied to the traffic sign and the HUD is chosen as the appropriate display.

Figure 8 displays the arrangement of the LED modules on the hood of the simulator vehicle. While the modules for both concepts are installed, the corresponding LED arrays are never used together at the same time.

\section{A. Concept 1: "Static Guiding Point"}

For this concept - derived from the first prototype - the LED array which is arranged between the edge of the front lid and the windshield is used. Consisting of 90 LEDs it has a length of $1.6 \mathrm{~m}$. It features the same color encoding as in the concept of dynamic guiding points along with a change in size of the guiding point. Additionally the point has no sharp edge but there is an exponential brightness degradation over 3 LEDs to create a smoother appearance. As the maximum distance to a deceleration target is $1000 \mathrm{~m}$, this corresponds to a point size of 3 LEDs in the middle of the array. This point increases linearly in its width up to the full array length when a distance of $0 \mathrm{~m}$ is reached. To be consistent with this behavior, the distance indicator in the HUD also has a linear scale. On activation the brightness is increased quadratic to the maximum with a time constant of $2 \mathrm{~s}$. For deactivation the inverse effect is being applied. Similar to the other concept the transition time between different colors is set to $2 \mathrm{~s}$. Figure 6 shows the impression of the installed LED lines from the driver perspective. All LEDs are simultaneously turned on to show the overall installation, both assistance concepts never create such an output.

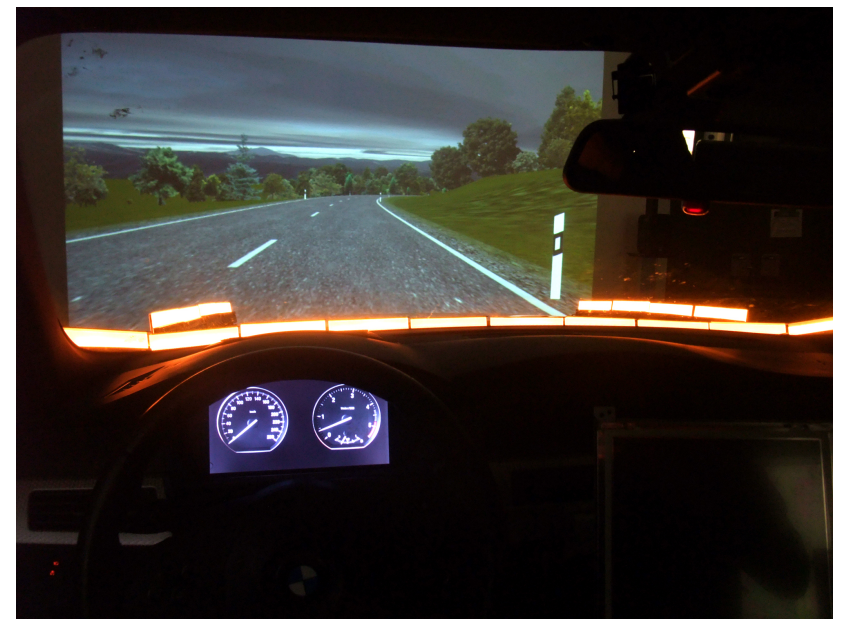

Fig. 6. Driver view on the second prototype, all modules switched on

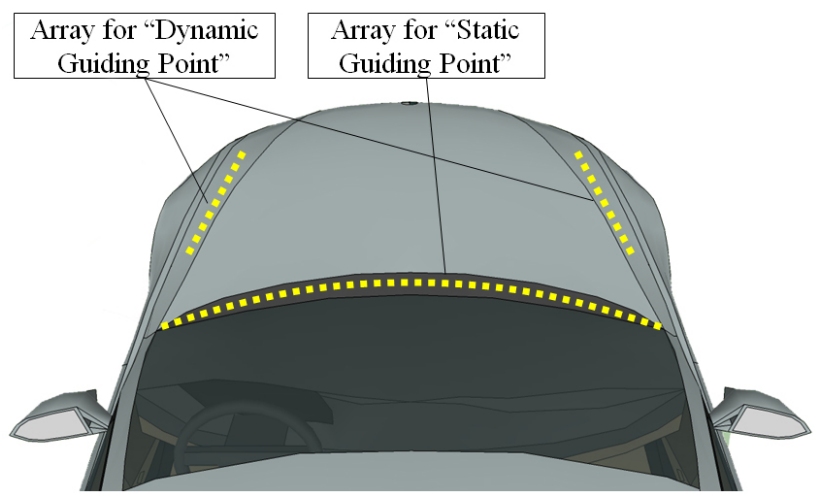

Fig. 7. Arrangement of LED arrays on front lid, seen from above

\section{B. Concept 2: "Dynamic Guiding Points"}

The second concept to adapt is described in [4]. The original installation was a lighting system mounted on the walls of a tunnel. Light dots were switched on and off in a way to create the impression of an array of dots moving along the tunnel with the optimal speed. It is mentioned that drivers tended to move with the same speed as the dots, but not how strong this effect was.

This idea shall now be moved to the car to have such a system always on board. As can be seen in figure 7, two symmetric diagonal lines with a length of $64 \mathrm{~cm}$ equipped with 36 LEDs per line are installed on the front lid. From the driver view these lines seem to be nearly parallel to the road course and are just below the primary field of view. The same algorithm which analyses the driving situation to suggest a deceleration strategy as described for the first prototype is employed here, too. Color coding of the dots will also be applied, but compared to the first prototype different colors are chosen. The encoding is as follows ( $\mathrm{RGB}$ color calibration, encoded in $(\mathrm{R}, \mathrm{G}, \mathrm{B})$ ):

- Coasting (motor drag torque): yellow $(255,255,0)$

- Light braking $(-0.2 \mathrm{~g})$ : orange $(255,140,0)$

- Strong braking $(-0.4 \mathrm{~g})$ : red $(255,0,0)$ 
It was also mentioned in the first evaluation that the transition time of $1 \mathrm{~s}$ between different colors is too short. For the following experiments a time constant of $2 \mathrm{~s}$ will be applied. In this concept the dots on the diagonal line arrays move away from the driver if the driver is too slow or towards the driver if he is too fast, which will usually be the case. The moving speed of the dots depends on the difference between the suggested and the actually driven speed, the higher the difference the faster is the dot movement. For calculating the dot speed the following equation is used:

$$
\Delta x_{\text {dot }}=0.04 \cdot \frac{v_{\text {ego }}-v_{\text {target }}}{\frac{k m}{h}} \text { with } \quad \Delta x_{\text {dot }} \in[0,1.5]
$$

It was found empirically with expert users that the coefficient 0.04 in equation 3 results in a comfortable dot speed. To calculate the "physical" speed of a dot, it must be known that a $\Delta x_{d o t}$ of 1 in the LED coordinate system equals a distance of $1.78 \mathrm{~cm}$ and that the update rate of the LED system is 30 Hz. A speed difference of $10 \frac{\mathrm{km}}{\mathrm{h}}$ would equal a $\Delta x_{\text {dot }}$ of 0.4 LEDs and thus a speed of $0.4 \cdot 1.78 \mathrm{~cm} \cdot 30 \mathrm{~Hz}=21.36 \frac{\mathrm{cm}}{\mathrm{s}}$.

Once the system is activated 3 static points fade to full brightness and start moving with the desired speed when fading is finished. This creates a smooth transition from the deactivated to the activated state. For deactivation the brightness is faded out while the points are moving.

\section{Experiment description}

A experiment with the two new concepts was conducted in a driving simulator experiment. There were 45 participants divided into three groups of 15 persons. The first group was used to gain reference data by driving without any assistance system. Participants of the second and third group were each given the support of one of the assistance concepts.

Additionally all participants of the last two groups had to answer a questionnaire about the respective concept. The procedure of the experiment was as follows:

- Test drive to get used to the simulator behavior

- Explanation of the assistance concept by the investigator

- Drive with assistance concept

- Questionnaire about the concept

All drives were conducted on similar courses which all included two traffic jams on a highway, several speed limits and two construction sites on a rural road. These situations stayed the same for every course, but were altered in sequence. By using an individual course for each participant of a group, learning effects should be minimized and comparability of situations be improved.

All participants were given a peripheral detection task (PDT) while driving to measure their concentration. Red dots were displayed in the driver's central and peripheral field of view. They were arranged in one of five equal distributed horizontal positions with a fixed vertical position. The time between two dots varied between 2 and $6 \mathrm{~s}$ while every dot was visible for a maximum time of $2 \mathrm{~s}$. Drivers had the task to acknowledge each dot by pressing a button on the steering wheel.
TABLE II

RATINGS OF CONCEPT 1 AND CONCEPT 2

\begin{tabular}{|l|l|l|l|l|}
\hline Question & Rating 1 & $\sigma$ 1 & Rating 2 & $\sigma 2$ \\
\hline $\begin{array}{l}\text { Would you use this dis- } \\
\text { play in your car? }\end{array}$ & 3.60 & 0.95 & 3.44 & 0.93 \\
\hline $\begin{array}{l}\text { Did you find it optically } \\
\text { appealing? }\end{array}$ & 3.73 & 0.85 & 3.63 & 1.05 \\
\hline $\begin{array}{l}\text { Could you understand it } \\
\text { intuitively? }\end{array}$ & 4.4 & 0.71 & 3.75 & 1.25 \\
\hline $\begin{array}{l}\text { Did it support anticipa- } \\
\text { tory driving? }\end{array}$ & 4.2 & 0.75 & 4.06 & 0.83 \\
\hline
\end{tabular}

\section{Results}

The questionnaire for this experiment was extended in respect of the first evaluation by a question about the need for the distance information in the Head-Up Display. All other questions remained the same and can therefore be compared to the first prototype.

The results in Table II show a significant $(P<0.01)$ improvement of the first two ratings and a marginally significant $(P<0.05)$ improvement for the last rating, determined with a Mann-Whitney U Test. Figure 8 also depicts a rating improvement for both new concepts. They are being judged by participants to be more pleasant and joyful while also being less disturbung, obtrusive and plump than the first prototype with a significant $(P<0.01)$ improvement.

As the activating logic behind the user interface is the same in all experiments with the same traffic situations, the differences in the ratings are likely to originate from the different optical output system and lighting effects. It can also be concluded that the adjustments based on the first experiment results produced the desired improvements in the user ratings.

\section{E. Results of the driving experiment}

Evaluation of the data collected during the driving experiments showed a considerable decrease of reaction times for drivers using one of the assistance concepts. Additionally they were able to reach corresponding speed minima of the reference group while travelling shorter distances.

Figure 9 illustrates the difference of reaction times. The reaction time shall be defined as the period between timestamp "0" and the time of maximum speed. While it's clearly visible that the total time required to reach the speed minimum is higher for drivers guided by the assistance system, the general course of speed decline is clearly more constant and seems to be almost linear.

For the other concept figure 10 shows the data recieved from the group driving with the dynamic guiding points while being confronted with a speed limit on a highway. Again a smooth decceleration is can be noticed when looking at the average assisted speed.

It can further be seen that the average speed of the baseline group doesn't reach the specified limit of $100 \mathrm{~km} / \mathrm{h}$. The minimum average speed of the reference group is reached almost $4 \mathrm{~s}$ earlier by the drivers with the dynamic guiding points. 




Fig. 8. Results of the semantic differential questions for the second prototype, results of first prototype are included as reference

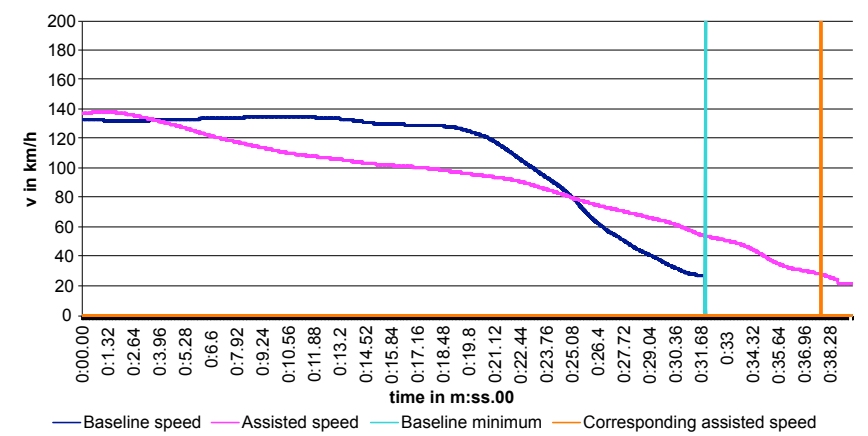

Fig. 9. Average speed developing over time for drivers with static guiding point assistance in a traffic jam situation

Table III shows the improvements in reaction time as well as differences of time and distance until the minimal baseline speed is reached. For this purpose the traffic jam situation and the speed limit situation are being analyzed.

\section{CONCLUSION AND FUTURE WORK}

We described two stages of development of a LED system which support anticipatory driving. With the first prototype a user study was conducted to obtain a first impression of suitable system parameters and arrangement of the LEDs. Based on this the second stage was implemented with two assistance

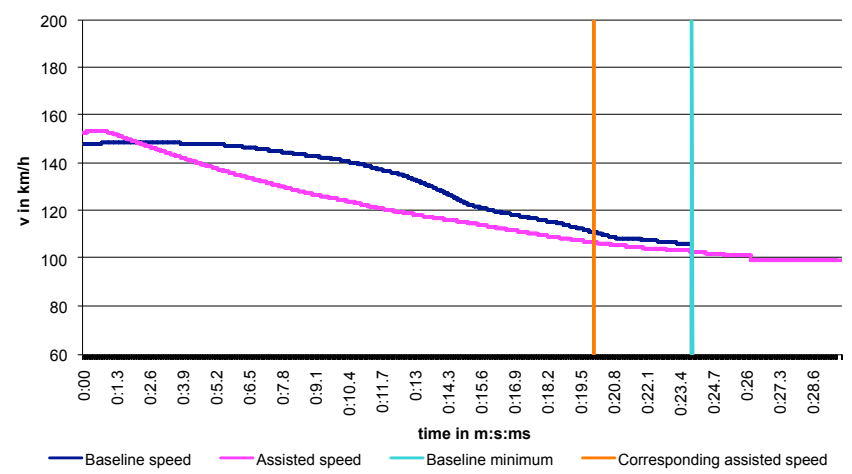

Fig. 10. Average speed developing over time for drivers with dynamic guiding points assistance in a speed limit situation

TABLE III

COMPARISON OF CONCEPTS FOR A TRAFFIC JAM AND SPEED LIMIT

\begin{tabular}{|c|c|c|c|c|}
\hline Situation & Participant Group & Reaction time & $\Delta \mathrm{t}$ & $\Delta \mathrm{x}$ \\
\hline \hline Traffic jam & Baseline & 12.24 & & \\
\hline & Static point & $1.57 \mathrm{~s}$ & $5.92 \mathrm{~s}$ & $-11.57 \mathrm{~m}$ \\
\hline & Dynamic points & $0.78 \mathrm{~s}$ & $10.14 \mathrm{~s}$ & $-2.12 \mathrm{~m}$ \\
\hline \hline Speed limit & Baseline & $5.09 \mathrm{~s}$ & & \\
\hline & Static point & $1.18 \mathrm{~s}$ & $-2.44 \mathrm{~s}$ & $-113.44 \mathrm{~m}$ \\
\hline & Dynamic points & $0.74 \mathrm{~s}$ & $-3.86 \mathrm{~s}$ & $-166.33 \mathrm{~m}$ \\
\hline
\end{tabular}

concepts. Both new concepts showed the desired impact of an earlier and more constant deceleration while receiving better acceptance judgements by the evaluation participants.

Next steps are additional user evaluations in a driving simulator to further assess the impact on driver behavior and consequently vehicle dynamics and fuel consumption. Also experiments in a real vehicle are planned to obtain an impression of the system effectiveness in a real setting.

\section{ACKNOWLEDGMENT}

We would like to thank Christoph Wierer for developing the circuit boards and building the initial version of the second prototype system. This work was also supported by BMW Forschung und Technik GmbH.

\section{REFERENCES}

[1] D. Rösler, J. F. Krems, S. Mahlke, M. Thring, K. Seifert, Evaluation of night vision enhancement systems: Driver needs and acceptance, In C. Stiller \& M. Maurer (Eds.). Fahrerassistenzsysteme (pp. 97-103). Karlsruhe: fmrt Karlsruhe e.V., 2006.

[2] D. Popiv, K. Bengler, M. Rakic, M. Duschl, F. Laquai, Reduction of fuel consumption by early anticipation and assistance of deceleration phases, Fisita World Automotive Congress, Budapest, 2010.

[3] D. Popiv, C. Rommerskirchen, K. Bengler, M. Duschl, M. Rakic, Effects of assistance of anticipatory driving on driver's behaviour during deceleration phases, Humanist Conference, Berlin, 2010.

[4] A. Zlocki, Verkehrsflussoptimierung mittels Lauflicht, Institut für Kraftfahrzeuge, Aachen, 2005.

[5] P. Pretto, A. Chatziastros, Changes in optic flow and scene contrast affect the driving speed, Driving Simulation Conference Europe (pp. 263-272), Paris, 2006.

[6] American National Standard, E1.11 - 2004 Asynchronous Serial Digital Data Transmission Standard for Controlling Lighting Equipment and Accessories, American National Standards Institute (ANSI), 2004. 